\title{
Fulminant Guillain-Barré Syndrome (GBS) with a Constellation of Uncommon Clinical Manifestations: Differential Diagnosis and Treatment Challenges
}

\author{
Youssef Ghosn ${ }^{1}$, Mohammed Hussein Kamareddine ${ }^{1}$, Hilal Abdessamad ${ }^{2}$, Tarek Safi ${ }^{1}$, Carmen Adem ${ }^{3}$, \\ Rosette Jabbour ${ }^{4}$ \\ ${ }^{1}$ Faculty of Medicine and Medical Sciences, University of Balamand, Beirut, Lebanon; Departments of ${ }^{2}$ Internal Medicine, \\ ${ }^{3}$ Radiology and ${ }^{4}$ Neurology, St. George Hospital University Medical Center and University of Balamand, Beirut, Lebanon.
}

\section{Corresponding Author:}

Dr. Rosette Jabbour

Email: rosette.jabbour@balamand.edu.lb

This is an Open Access article distributed under the terms of the Creative Commons Attribution License (creativecommons.org/ licenses/by/3.0).

Received : February 26, 2019

Accepted : July 8, 2019

Published : July 30, 2019

\begin{abstract}
Background: Fulminant Guillain-Barré syndrome (GBS) could present with myriad of clinical findings requiring an aggressive, yet under-investigated, treatment for case resolution. Case Report: We herein discuss a case of fulminant Guillain-Barré syndrome (GBS) in a previously healthy 25-year-old female patient presenting for ascending limb weakness and paraesthesia. The nerve conduction studies (NCS) and electromyography (EMG) with the magnetic resonance imaging (MRI) of the spinal cord were all consistent with a diagnosis of GBS. The patient gradually developed quadriplegia and facial diplegia despite treatment. In addition, she had severe sensory disturbances that consisted mainly of paraesthesia, dysesthesias, neuropathic pain and anaesthesia. Few days later she developed type II respiratory failure, requiring urgent intubation and intensive care unit (ICU) transfer despite treatment. Moreover, she had autonomic dysfunction consisting of tachycardia and bradycardia, nausea, urine and fecal incontinence as well as diaphoresis and syndrome of inappropriate antidiuretic hormone secretion (SIADH). Conclusion: In view of the severe clinical course of this disease, the patient was initially treated with two cycles of intravenous IgG immunoglobulin (IVIG) administration followed by five sessions of plasmapheresis. Marked clinical improvement was noticed after the $4^{\text {th }}$ session of plasmapheresis.
\end{abstract}

Keywords: Electromyography, Immunoglobulin, Paresthesia, Plasmapheresis, Quadriplegia, Spinal Cord.

\section{Introduction}

The presence of a constellation of both common and uncommon GBS findings in one clinical setting made this case both unique and challenging. Additionally, the fact that the symptoms did not start to resolve before the $4^{\text {th }}$ session of plasmapheresis and after two cycles of intravenous immunoglobulin (IVIG) raises questions regarding what treatment modality is beneficial in severe and fulminant case of GBS.

\section{Case Report}

A 25-year-old female patient with no previous medical history, presented for lower limb weakness and paresthesia. The history goes back to three weeks prior to her presentation when she developed gastritis which was self-limiting. Two days prior to her presentation, she had developed an "unpleasant sensation" in her lower limbs that quickly evolved to lower limbs tingling and motor weakness associated with lower back pain and bilateral radicular pain. The weakness started distally and gradually worsened and ascended proximally to the lower limbs and the upper limbs.

The neurological examination upon presentation showed distal and proximal weakness of $1 / 5$ motor power in the lower extremities and $4 / 5$ in the upper extremities. Proprioception and 
vibration sensation were affected in all limbs. Diffuse allodynia was present all over. The deep tendon reflexes (DTRs) were absent in four limbs with negative Babinski signs bilaterally. No cranial nerves involvement was noticed and the rest of the general physical exam was normal. She was afebrile, normotensive, her heart rate was 60 beats/ min and the oxygen saturation $\left(\mathrm{SpO}_{2}\right)$ on room air was $98 \%$.

Upon admission, nerve conduction study (NCS) and electromyogram (EMG) showed prolonged distal motor latencies in the peroneal and posterior tibial nerves bilaterally. F-wave responses were prolonged, raising the diagnosis of Guillain-Barré syndrome (GBS). The MRI of the spinal cord showed homogeneous enhancement in the anterior and posterior roots of the spinal nerves [Fig.1], findings favoring also the diagnosis of GBS. Laboratory tests showed no significant findings. IgA levels were normal. Serologies were negative for brucella titers, human immunodeficiency virus (HIV) and Venereal Disease Research Laboratory test (VDRL). One-week post admission, a lumbar puncture (LP) was done and the cerebrospinal fluid (CSF) analysis showed: 2 red blood cells (RBCs), 4 white blood cells (WBCs) $/ \mathrm{mm}^{3}$, proteins level of $656 \mathrm{mg} / \mathrm{dL}$, glucose level of $68 \mathrm{mg} / \mathrm{dL}$. Herpes viral panel polymerase chain reaction (PCR), toxoplasma PCR, epstein barr virus (EBV) PCR, cytomegalovirus (CMV) PCR were all negative.

The differential diagnosis of a 25-yearold female presenting with motor weakness and dysesthesia in all four limbs is broad. In this patient, the clinical findings could be due to an infection, autoimmune disease, mass/neoplastic effect, psychiatric etiologies or toxin induced. Infectious causes known to mimic GBS include Lyme's disease, HIV infection, EBV and CMV [1]. Additionally, we investigated possible causes of encephalitis and meningoencephalitis including herpes, toxoplasma [2], neuro-brucellosis [3], in addition to neurosyphilis. Mass effect could be due to leptomeningeal malignancy and acute

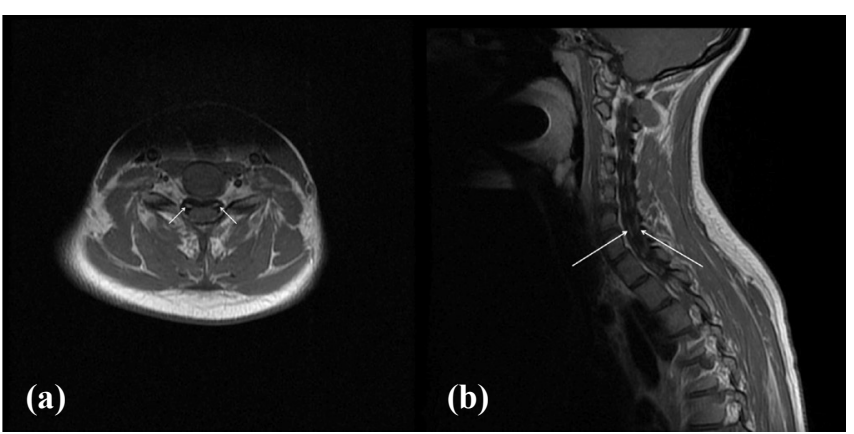

Fig.1: Spinal MRI: axial (a) and sagittal (b) T1 post IV contrast administration. Homogeneous enhancement is seen on anterior (a and $\mathbf{b})$ and posterior (b) nerve roots (white arrows), in favour of GBS.

myelopathy (lumbar disc stenosis or transverse myelitis) [1,4]. Autoimmune causes include multiple sclerosis (MS), myasthenia gravis [1] and acute onset of chronic inflammatory demyelinating polyneuropathy (CIDP) [5]. Toxin exposure include botulinum and heavy metal toxicity $[1,4]$. Other causes include vascular insults [4]. Moreover, conversion disorder/ hysterical paralysis should be considered as diagnosis of exclusion [6]. Lyme was excluded because Lebanon is not an endemic region and the patient denies any travel history. PCR was negative for herpes, EBV, toxoplasma and CMV. Serology ruled out HIV, brucella and syphilis. CIDP was also ruled out in this patient because the neurological symptoms started two days prior to presentation, and CIDP diagnosis requires at least 8 weeks of persistent symptoms. Normal imaging and the fast-progressive paralysis made idiopathic intracranial hypertension and CNS neoplasia unlikely. Brain and spine MRIs helped in excluding MS lesions and did not reveal spinal cord compression, radiculopathy, tumors or abscesses. Transverse myelitis generally has a well localized, clearly defined sensory level involvement and does not present as progressive ascending weakness. Vascular insults were excluded because the patient had no risk factors and the fast-progressive presentation with normal lab findings is highly unlikely in the setting of a vascular injury. Myasthenia gravis has no sensory symptoms and changes in intensity with 
daily activity which is not the case here. No risky behaviours associated with botulism were identified and the absence of descending paralysis makes the diagnosis unlikely. Lead and mercury exposure were ruled out because they present with slowly progressive weakness more common in the upper limbs [4] and the history did not indicate exposure to these substances. Negative history of psychiatric illnesses and the progression of the patient's presentation made conversion disorder unlikely. EMG, MRI and LP (performed one week post presentation) findings showed findings in favour of GBS, including prolonged distal motor latencies with F-wave prolongation, anterior and posterior root enhancement and acellularity with high proteins, respectively.

The treatment administered to the patient consisted of two sessions of IVIG (the first course was $24 \mathrm{~g} / \mathrm{d}$ and the second $30 \mathrm{~g} / \mathrm{d}$ ) for 5 days each with no resolution. This was followed by 5 sessions of plasmapheresis which were started 16 days after last IVIG. Pregabalin, pethidine, amitriptyline and duloxetine were given for neuropathic pain control. Midazolam and fentanyl were given as sedation prior to and during patient intubation. Regarding the dysautonomia symptoms, the patient was started on amiodarone and bisoprolol for the management of tachycardia, and was put on water restriction for the management of SIADH. Additionally, she developed hypocalcemia secondary to plasmapheresis which was managed with intravenous (IV) calcium gluconate.

On the floor, the patient had massive paralysis of her lower extremities which progressed to include her upper extremities, with intense dysesthesia affecting her face and all her limbs. She was given IVIG (Sandoglobulines) $0.4 \mathrm{~g} / \mathrm{kg} /$ day $(24 \mathrm{~g} / \mathrm{d})$ for 5 days. On day 3 of IVIG, her pain increased so pregabalin was started and a full dose was given. Since her pain did not resolve, pethidine was then added. However, few days after completion of the first course of IVIG, she became dyspneic. The patient's arterial blood gases $(\mathrm{ABG})$ showed carbon dioxide $\left(\mathrm{CO}_{2}\right)$ retention necessitating her transfer urgently to the ICU for intubation secondary to type II respiratory failure. The following day, the patient became totally quadriplegic and developed facial diplegia along with diffuse body dysesthesia. She was started on fentanyl for pain management and light sedation and given a second course of IVIG, $30 \mathrm{~g} / \mathrm{d}$ for 5 days. However, despite treatment, some features of dysautonomia occurred, consisting of rapidly alternating tachycardia reaching up to $170 \mathrm{BPM}$ and bradycardia down to 40 BPM, urine and faecal incontinence, and profuse diaphoresis. In addition, she developed SIADH decreasing sodium levels as low as $120 \mathrm{mg} / \mathrm{dL}$. Her symptoms did not improve after the second course of IVIG. One week later, her motor strength in her arms improved to +2 , she was switched to continuous positive airway pressure (CPAP) support with good tolerance and was extubated the following day. However, few hours later, the patient failed the extubation and was reintubated again for respiratory distress. This was followed by worsening of her neurological status leading to complete quadriplegia. Subsequently, plasma exchange therapy was started after 16 days of the $2^{\text {nd }}$ course of IVIG and the patient had undergone tracheostomy. Following completion of plasma exchange therapy, the patient was weaned off ventilation, successfully. She was stable and her motor strength improved to $3 / 5$ in the upper limbs and $1 / 5$ in the lower limbs. The dysesthesia became minimal as well as the autonomic symptoms. As a consequence, she was transferred out of the ICU to a regular floor.

A repeat NCS and EMG were performed at the completion of the fifth session of plasmapheresis and showed recruitment of some normal motor units, potentials activity and marked slowing in the conduction velocity of all nerves. On the floor, the patient was showing day-by-day improvement in her motor power on physical therapy. Her sensation became normal. She had a fenestrated tube inserted into her tracheostomy tube which was well-tolerated and removed completely thereafter. 
Oral intake was resumed progressively and was well-tolerated. She was eventually discharged to a rehabilitation center to continue her long-term motor re-education.

\section{Discussion}

What makes this case interesting is the presence of myriad of GBS findings including uncommon ones in addition to the aggressive treatment that was required for case resolution. Throughout the course of the disease progression, the patient had bilateral limb sensory disturbances consisting mainly of paraesthesia, dysesthesias and anaesthesia. Bilateral motor symptoms ranging from weakness to complete paralysis, facial diplegia and respiratory failure were also observed. Additionally, the patient had autonomic dysfunction consisting of tachycardia, bradycardia, nausea, diaphoresis, urinary and faecal incontinence and SIADH. The severity of the case required two cycles of IVIG administration followed by plasmapheresis to achieve clinical stabilization.

With regard to the prevalence of various clinical manifestation of GBS, data shows that 20$30 \%$ of GBS patients experience respiratory failure [1], approximately $50 \%$ of patients experience severe pain [7], and $60-70 \%$ have autonomic dysfunction symptoms [8]. Anandan C et al. investigated the prevalence of various autonomic dysfunctions which showed that diarrhoea/ constipation present in $15.5 \%$ of the cases, hyponatremia in $14.9 \%$, SIADH in $4.8 \%$, bradycardia in $4.7 \%$ tachycardia $2.7 \%$, sweating disturbances in $0.1 \%$ and urinary retention in $3.9 \%$ of cases [9]. Our patient had SIADH, alternating episodes of bradycardia and tachycardia in addition to diaphoresis. Facial diplegia is a relatively common manifestation in GBS patients with it being reported in around 30\%-50\% of cases [8]. Our patient had facial diplegia with dysesthesias. To our knowledge, the prevalence of facial dysesthesias is still unknown. Finally, it is generally accepted that IVIG treatment and plasmapheresis have the same efficacy and that combining regiments is no better than the use of a single treatment modality [10]. This concept seems to be challenged in cases of severe fulminant GBS such as this case. Kamei A et al. discussed a case of paediatric fulminant GBS which did not resolve prior to the initiation of plasmapheresis which was then followed by IVIG administration [11]. The same problem was presented by Revathy et $\mathrm{al}$. who discussed a case of fulminant GBS in a 56-year-old male refractory to IVIG which required 14 sessions of plasma exchange [12]. Although treatment regimens including IVIG alone, plasma exchange alone or various combinations of IVIG and plasma exchange were found to be successful in a number of other cases of fulminant GBS [13-18], there is no randomized control trials that investigate the efficacy of using different treatment modalities when patients with GBS continue to deteriorate after plasma exchange or a standard course of IVIG alone [19]. The fact that many patients do not improve until both IVIG and plasmapheresis have been administered raises doubts regarding the treatment regimen in severe cases of GBS with various clinical manifestations.

\section{Conclusion}

Fulminant cases of GBS can have a variety of clinical manifestations and subsequent complications. Although, plasma exchange therapy and IVIG have the same efficacy in the treatment of GBS and could be used interchangeably, in this case report, improvement of the symptoms was not achieved until both treatments were combined. More studies should focus on the importance of treating severe and fulminant cases of GBS with both IVIG and plasma exchange therapy.

Contributors: YG: suggesting writing this article and manuscript writing; MHK: reviewing the literature and manuscript writing. $\mathrm{HA}, \mathrm{RJ}$ : critical inputs into the manuscript. TS: manuscript editing. CA: interpreting the imaging studies of this article, manuscript editing. RJ will act as a study guarantor. All authors approved the final version of this manuscript.

Funding: None; Competing interests: None stated. 


\section{References}

1. Willison HJ, Jacobs BC, Van Doorn PA. Guillain-barre syndrome. The Lancet. 2016;388(10045):717-727.

2. Granerod J, Ambrose HE, Davies NW, Clewley JP, Walsh AL, Morgan D, et al. Causes of encephalitis and differences in their clinical presentations in England: a multicentre, population-based prospective study. The Lancet infectious diseases. 2010;10(12):835-844.

3. Goktepe AS, Alaca R, Mohur H, Coskun U. Neurobrucellosis and a demonstration of its involvement in spinal roots via magnetic resonance imaging. Spinal cord. 2003;41(10):574.

4. Randall DP. Guillain-Barré syndrome differential diagnosis. Disease-a-Month. 2010;56(5):266-278.

5. Ruts L, Drenthen J, Jacobs BC, Van Doorn PA, Dutch GBS Study Group. Distinguishing acute-onset CIDP from fluctuating Guillain-Barré syndrome a prospective study. Neurology. 2010;74(21)1680-1686.

6. Wherry JN, McMillan SL, Hutchison HT. Differential diagnosis and treatment of conversion disorder and Guillain-Barre syndrome. Clinical Pediatrics. 1991;30(10):578-585.

7. Levin KH. Variants and mimics of GuillainBarré syndrome. The Neurologist. 2004;10(2):61-74.

8. Walling AD, Dickson, G. Guillain-Barré syndrome. American Family Physician. 2013;87(3);191-197.

9. Anandan C, Khuder SA, Koffman BM. Prevalence of autonomic dysfunction in hospitalized patients with Guillain Barré syndrome. Muscle \& Nerve. 2017;56(2):331-333

10. Hadden RDM, Cornblath DR, Hughes RA, Zielasek J, Hartung HP, Toyka KV, Swan AV, Plasma exchange/ Sandoglobulin Guillain-Barré syndrome trial group. Electrophysiological classification of Guillain-Barré syndrome: Clinical associations and outcome. Ann Neurol. 1998;44(5):780-788.
11. Kamei A, Akasaka M, Araya N, Ishikawa K, Takada A, Furukawa $\mathrm{H}$, et al. Successful management of fulminant Guillain-Barré syndrome and its complications. Pediatric Emergency Care. 2018;34(5):e87-e89.

12. Revathy N, Abhinav R, Muralidharan N, Debasish G. Fulminant Guillain Barré syndrome (GBS) refractory to intravenous immunoglobulin (IVIG) treated with therapeutic plasma exchange. Hematol Transfus Int J. 2017;5(3):00120.

13. Rougé A, Lemarie J, Gibot S, Bollaert PE. Long-term impact after fulminant Guillain-Barré syndrome, case report and literature review. International Medical Case Reports Journal. 2016;9:357.

14. Sevketoglu E, Tatlı B, Tuğcu B, Demirelli Y, Hatipoglu S. An unusual cause of fulminant Guillain-Barre syndrome: angel's trumpet. Pediatric Neurology. 2010;43(5),368370.

15. Bohlega SA, Stigsby B, Haider A, McLean D. Guillain Barré syndrome with severe demyelination mimicking axonopathy. Muscle \& Nerve. 1997;20(4):514-516.

16. Moussouttas M, Chandy D, Dyro F. Fulminant acute inflammatory demyelinating polyradiculoneuropathy. Neurocritical Care. 2004;1(4):469-473.

17. Tagami S, Susuki K, Takeda M, Koga M. Fulminant case of Guillain Barré syndrome with poor recovery and depression following Haemophilus influenzae infection. Psychiatry and Clinical Neurosciences. 2008;62(4):486486.

18. Medici C, Gonzalez G, Cerisola A, Scavone C. Lockedin syndrome in three children with Guillain-Barré syndrome. Pediatric Neurology. 2011;45(2):125-128.

19. Meena AK, Khadilkar SV, Murthy JMK. Treatment guidelines for Guillain-Barré syndrome. Annals of Indian Academy of Neurology. 2011;14(Suppl1):S73. 